\title{
Photoreceptor inner segment ellipsoid band integrity on spectral domain optical coherence tomography
}

This article was published in the following Dove Press journal:

Clinical Ophthalmology

9 December 2014

Number of times this article has been viewed

\author{
Sandeep Saxena' \\ Khushboo Srivastav' \\ Chui M Cheung ${ }^{2}$ \\ Joanne $\mathrm{YW} \mathrm{Ng^{3 }}$ \\ Timothy YY $\mathrm{Lai}^{3}$ \\ 'Retina Service, Department of \\ Ophthalmology, King George's \\ Medical University Lucknow, \\ India; ${ }^{2}$ Singapore National Eye \\ Centre, Singapore; ${ }^{3}$ Department \\ of Ophthalmology and Visual Sciences, \\ The Chinese University of Hong Kong, \\ Kowloon, Hong Kong
}

\begin{abstract}
Spectral domain optical coherence tomography cross-sectional imaging of the macula has conventionally been resolved into four bands. However, some doubts were raised regarding authentication of the existence of these bands. Recently, a number of studies have suggested that the second band appeared to originate from the inner segment ellipsoids of the foveal cone photoreceptors, and therefore the previously called inner segment-outer segment junction is now referred to as inner segment ellipsoidband. Photoreceptor dysfunction may be a significant predictor of visual acuity in a spectrum of surgical and medical retinal diseases. This review aims to provide an overview and summarizes the role of the photoreceptor inner segment ellipsoid band in the management and prognostication of various vitreoretinal diseases.

Keywords: spectral domain optical coherence tomography, inner segment-outer segment junction, external limiting membrane, macular hole, diabetic macular edema, age relate macular degeneration
\end{abstract}

\section{Introduction}

Optical coherence tomography (OCT) is a powerful imaging tool that can be used for cross-sectional imaging of biological tissues. The relatively thin and transparent retina makes OCT an ideal method for ocular imaging. It can be used for visualization and delineation of the retinal cell layers to detect and monitor a variety of retinal diseases. Nearly 2 decades since its introduction, OCT has become an indispensable tool for research, screening, diagnosing, and monitoring diseases of the macula and optic nerve head.

OCT imaging technology has been evolving rapidly. Rapid acquisition speed of spectral domain (SD) OCT provides multiple advantages including reduced motion artifacts, improved transverse sampling, and increased axial resolution. These factors enable production of more detailed images and accurate portrayal of the true retinal contour.

Within the outer retina, a hyperreflective band previously thought to represent the photoreceptor inner segment/outer segment (IS/OS) junction can be appreciated on SD-OCT. Integrity of this layer has been found to be of high clinical importance as absence or disruption of this layer has been found to be associated with various diseases affecting the photoreceptors and/or choroid. ${ }^{1-7}$

\section{Anatomical considerations}

In the foveal region, SD-OCT imaging of the macula has conventionally been resolved into four bands (Figure 1). The innermost and faintest band (first band) is believed to 


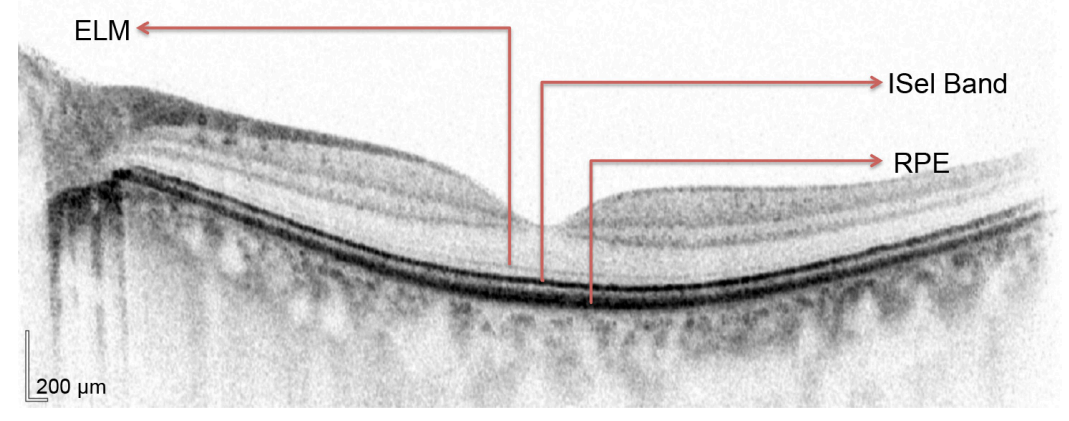

Figure I Normal Heidelberg spectral domain optical coherence tomography (SD-OCT) with intact external limiting membrane (ELM), photoreceptor inner segment ellipsoid band (ISel), and retinal pigment epithelium (RPE).

originate from the external limiting membrane $(\mathrm{ELM}),{ }^{8}$ which is an assembly of junctional complexes between Muller cells and photoreceptors. The second band is often referred to as the IS/OS boundary of the photoreceptors. ${ }^{9,10}$ The third band has been referred to as cone outer segment tips (COST) ${ }^{11}$ intermediate line, ${ }^{12}$ or as the Verhoeff membrane. ${ }^{13,14}$ The fourth band is supposed to represent the retinal pigment epithelium (RPE). Spaide and Curcio suggested that part of the fourth band is contributed by the Bruch's membrane and the choriocapillaris. ${ }^{15}$

However, some questions were raised regarding authentication of the existence of these bands. As shown in several studies, ${ }^{8,16-19}$ the length of the inner segment and outer segment was found to be approximately the same for both foveal rods and cones. From this, it can be deduced that IS/OS junction should lie at the midpoint between the ELM and the RPE. However, in normal SD-OCT, the second reflective band lies much closer to the ELM than the RPE. These doubts about the second band observed on SD-OCT were subsequently clarified. Fernandez et al demonstrated the anatomy of human foveal cone photoreceptors on SD-OCT and suggested that they visualized the inner segment ellipsoid band. ${ }^{20}$ Spaide and Curcio constructed a scale model drawing of the outer retina based on published histology to enable visual comparisons. ${ }^{15}$ Using this model for analysis, their conclusion on the origins of the first and fourth bands was the same as previously suggested. However, their analysis about the origin of the second band suggested that it originated from the inner segment ellipsoids. The third band seemed to have originated from the posterior tip of the cone outer segment ie, the contact cylinder. Lu et al used line scan OCT on excised living leopard frog retina to establish the origin of the existence of the IS/OS junction..$^{21}$ They speculated that the inner segment ellipsoid may contribute to the IS/OS junction in OCT images.
In this review, considering the evidence put forward by the above landmark studies, IS/OS junction will be referred to as inner segment ellipsoid (ISel), and various studies that assessed the role of the ISel band in SD-OCT in the diagnosis and prognosis evaluation of various surgical and medical retinal diseases will be discussed. Developments in several areas of OCT research promise exciting advances in performance and clinical applications. Adaptive optics uses deformable elements that corrects ocular aberrations and improves transverse resolution by decreasing spot size of the sample beam on the retina. ${ }^{22}$ Coupled with OCT, isotropic cellularresolution imaging may be achieved, which may be useful for studying photoreceptor morphology including ISel band in greater detail. This will further enhance our understanding of the role of ISel band in various retinal diseases. ${ }^{23}$

\section{Inner segment ellipsoid in surgical retinal diseases \\ Idiopathic macular hole}

Ultra high resolution OCT has enabled imaging of previously undetectable changes in photoreceptor ISel band associated with macular hole pathology and its post-operative repair (Figure 2). Macular hole reparative process was studied

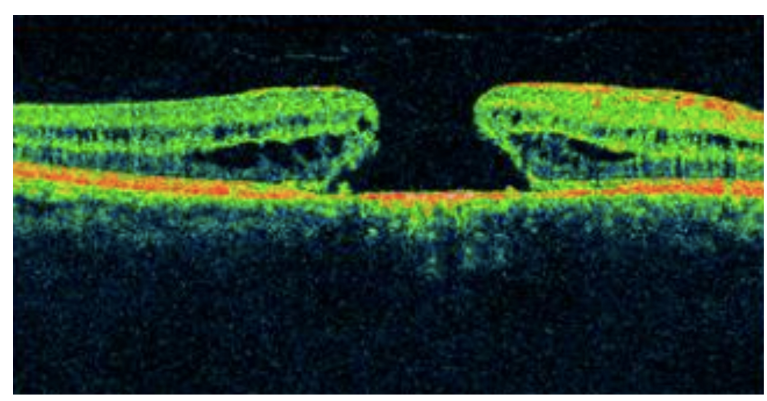

Figure 2 Macular hole with disrupted ISel and ELM. Abbreviations: ISel, inner segment ellipsoid; ELM, external limiting membrane. 
using SD-OCT in various studies and correlation analysis with best corrected visual acuity (BCVA) was performed. Vitrectomy, internal limiting membrane (ILM) peeling and internal gas tamponade is the standard treatment for achieving closure of macular hole. The reparative process of macular hole closure using SD-OCT was evaluated retrospectively and better visual outcome was observed in eyes with a continuous photoreceptor ISel band compared with those with a disrupted ISel band. ${ }^{24}$ In another study, Oh et al evaluated photoreceptor ISel band defect parameters in linear and composite SD-OCT formats, assessing diameter and area of the ISel band defect and macular density. ${ }^{4}$ They concluded that BCVA correlated better with the area of the photoreceptor ISel band defect than with linear measurements after macular hole closure.

Visualization of the ISel band was demonstrated to be an important indicator of photoreceptor integrity for both standard resolution and ultra high resolution OCT images. ${ }^{9}$ Thereafter, many studies were conducted to establish a relationship between the photoreceptor ISel band and BCVA in patients undergoing macular hole surgery. Presence of a normal photoreceptor ISel band was associated with better BCVA after surgery. ${ }^{25}$ The external limiting membrane was the first structure to recover after macular hole closure. ${ }^{26,27}$ During follow-up, foveal cysts may develop, which may later fill, leading to formation of an intact photoreceptor ISel band. ${ }^{27}$ Integrity of the ELM is directly correlated with restoration of the photoreceptor ISel band..$^{28}$

In further studies, even more detailed analysis was performed. The SD-OCT characteristics evaluated included macular hole diameter, ELM defect diameter, photoreceptor ISel band defect diameter, the presence or absence of subretinal fluid (SRF), central foveal thickness (CFT), and outer foveal thickness (defined as the distance between the ELM and the inner border of the RPE). Parameters that best correlated with BCVA postoperatively were outer foveal thickness and the photoreceptor ISel band respectively. Disrupted ELM was associated with poor BCVA. Outer foveal thickness gradually increased during the follow-up period. The results suggested that increase in outer foveal thickness indicates restoration of photoreceptor ISel band integrity, which is associated with better postoperative visual acuity. ${ }^{29,30}$

A recent retrospective study demonstrated that distinct a COST line was first seen at 6 months after the surgery. Moreover, in eyes with an intact photoreceptor ISel band and ELM, a distinct or irregular COST line had significantly better BCVA at 12 months compared with those with a disrupted COST line. It was concluded that appearance of a distinct or irregular COST line indicates recovery of foveal photoreceptor microstructure. ${ }^{31}$

A meta-analysis on 1,654 eyes with macular holes treated by pars plana vitrectomy revealed that $87.5 \%$ of eyes achieved anatomical closure and $12.5 \%$ failed to do so. ${ }^{32}$ Therefore, persistence of macular holes is still the major concern after macular hole surgery and further refinement of the surgical technique to enhance closure of macular holes is needed. Nonetheless, we should not forget to also focus our efforts on the analysis of predictive factors in determining not only surgical but also functional success in order to better advise our patients concerning their visual prognosis.

\section{Idiopathic epiretinal membrane}

Epiretinal membranes (ERMs) are collections of collagenous cells that occur on the inner surface of the central retina. These membranes have contractile properties and can lead to visual changes and metamorphopsia because of their effects on the underlying retina. ERMs can be treated with microincisional vitrectomy surgery and membrane peeling. However, visual acuity might not fully recover even after complete removal of an ERM. OCT is particularly useful in assessing the thickness and points of adhesions of the ERM in the macula (Figure 3). Various studies were conducted to assess the predictive factors of good BCVA postoperatively. An intact photoreceptor ISel band on SD-OCT represents morphological and functional recovery of photoreceptors and was associated with good BCVA after surgery. ${ }^{33}$ A disrupted photoreceptor ISel band was associated with poorer BCVA, and this disruption was found to be irreversible. Therefore, in patients with a disrupted photoreceptor ISel band, early removal of the ERM was warranted. ${ }^{34}$ Other studies also suggested that the presence or absence of a photoreceptor ISel band together with central retinal thickness, ILM profile, and foveal contour on SD-OCT are good predictors of BCVA postoperatively. ${ }^{35,36}$ Inoue et al suggested that with a preoperative intact photoreceptor

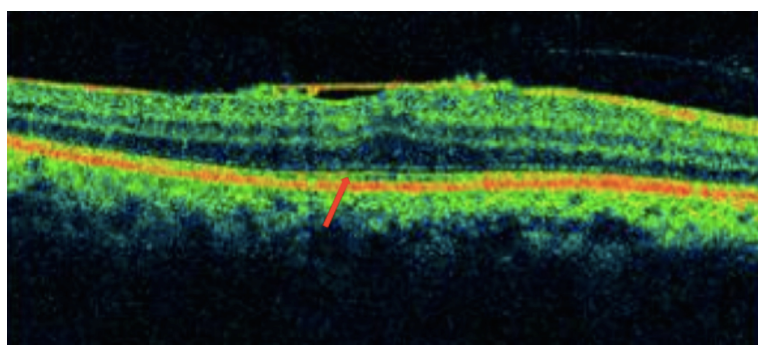

Figure 3 Epiretinal membrane showing photoreceptor ISel disruption (red arrow) Abbreviation: ISel, inner segment ellipsoid. 
ISel band, recovery of this band can occur over a follow-up period of 1 year after surgery. ${ }^{5}$ Preoperative integrity of the photoreceptor ISel band may be an important prognostic factor for better visual recovery after surgery. In another retrospective case series study on patients with secondary ERM after retinal detachment surgery, it was found that integrity of the photoreceptor ISel band and ELM were the main factors that predict final BCVA after ERM peeling. ${ }^{37}$ Disrupted photoreceptor ISel bands found preoperatively with normal autofluorescence can be associated with recovery of photoreceptor ISel bands following ERM peeling. It can be explained by the presence of a functional retinal pigment epithelium-photoreceptor complex, supporting normal photoreceptor activity. ${ }^{38}$

Based on several retrospective observational and interventional studies, ${ }^{39-42}$ it was established that integrity of the photoreceptor ISel band is correlated with BCVA. A number of studies also suggested that in addition to the photoreceptor ISel band and the COST line, photoreceptor outer segment length can also be considered to be an important prognostic factor for predicting BCVA postoperatively. ${ }^{40,41,43}$ Degree of metamorphopsia correlated with inner nuclear layer thickness in patients with ERM. ${ }^{39}$ Significant predictors for postoperative metamorphopsia outcome included the degree of preoperative metamorphopsia, central foveal thickness, and the photoreceptor ISel band integrity at baseline. ${ }^{44}$ Recently, it was concluded that photoreceptor ISel band restoration in the parafoveal quadrants also contributed significantly to the recovery of BCVA following ERM surgery in patients with metamorphopsia. Therefore, functional and morphological tests of the macular area should not be limited to the fovea but should be extended to the parafoveal region.

\section{Retinal detachment}

A retrospective observational study on patients with poor visual acuity after retinal detachment (RD) surgery demonstrated disruption of photoreceptor ISel bands on ultrahigh resolution OCT and confirmed the prior histological finding of damage to photoreceptor outer segments occurring after RD. ${ }^{45}$ Preoperative macula off RDs, the integrity of photoreceptor ISel bands and ELM signals on SD-OCT appeared to correlate with postoperative visual recovery. ${ }^{46-48}$ Preservation of the ELM postoperatively may predict the subsequent restoration of the photoreceptor layer. ${ }^{46}$ Preoperative intraretinal separation and outer retinal undulation was associated with higher incidence of disrupted photoreceptor ISel bands postoperatively. ${ }^{49}$ After correlating the OCT results with anatomic and functional outcome after scleral buckling surgery, it was

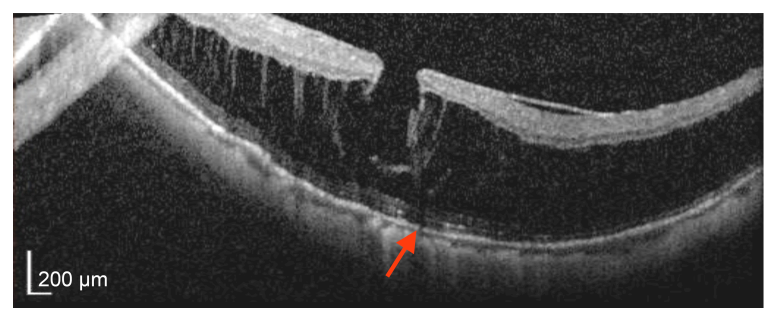

Figure 4 Myopic foveoschisis showing disruption of ISel band (red arrow) and ELM. Abbreviations: ISel, inner segment ellipsoid; ELM, external limiting membrane.

concluded that postoperative visual outcome was associated with the integrity of the photoreceptor ISel band..$^{50}$

\section{Myopic foveoschisis and foveal retinoschisis}

Myopic foveoschisis affects highly myopic patients with posterior staphyloma, and patients may later develop reduced vision due to foveal detachment and macular hole. SD-OCT imaging can demonstrate the characteristic splitting of retina with intraretinal bridges in myopic foveoschisis (Figure 4). In a study conducted to correlate the pathologic features of eyes with myopic foveoschisis with findings of SD-OCT, defects in photoreceptor ISel bands were found. ${ }^{51}$ In our study of two cases of bilateral X-linked foveal retinoschisis of different age groups, SD-OCT findings suggested disruption of the photoreceptor ISel band along with the outer nuclear layer and ELM disruption in the subfoveal region. ${ }^{52}$ Several surgical options have been proposed, which include pars plana vitrectomy with or without ILM peeling (traditional or fovea sparing), gas tamponade as well as scleral and suprachoroidal buckling techniques. The SD-OCT findings of an observational case study on patients with myopic foveoschisis undergoing vitrectomy suggested that postoperative visual acuity depended on recovery of the photoreceptor ISel band..$^{53}$

\section{Inner segment ellipsoid in medical retinal diseases \\ Age-related macular degeneration}

SD-OCT is currently the modality of choice for diagnosis and monitoring of neovascular age-related macular degeneration (AMD), as it is noninvasive and provides high resolution imaging (Figures 5 and 6). Depending on the layer of choroidal neovascularization (CNV) involvement, neovascular AMD can be divided into several subtypes. Type I lesions have CNV beneath the RPE corresponding to occult lesions in fluorescein angiography (FA). Type II lesions are located beneath the retina but above the RPE corresponding to classic lesions in FA. SD-OCT helps in differentiating between the two types of lesions. Type III CNV includes 


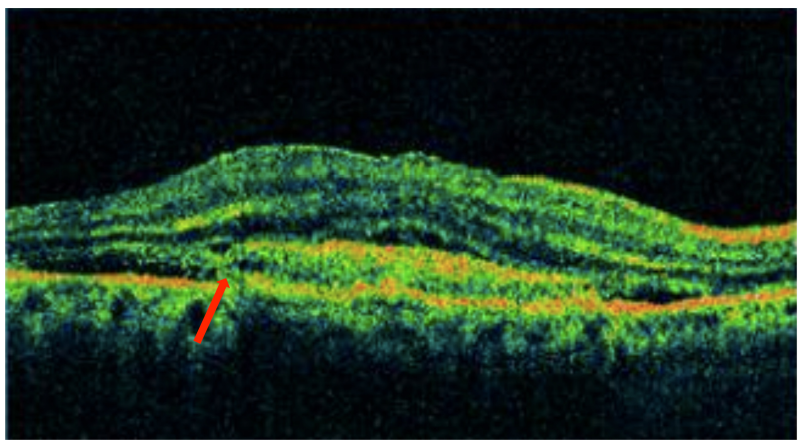

Figure 5 Choroidal neovascular membrane showing disruption of the photoreceptor ISel band, ELM, and RPE (red arrow).

Abbreviations: ISel, inner segment ellipsoid; ELM, external limiting membrane; RPE, retinal pigment epithelium.

retinal angiomatous proliferation and polypoidal choroidal vasculopathy (PCV) ${ }^{54}$ Microperimetry, SD-OCT, and fundus autofluorescence (FAF) have been used for evaluation of patients with progressive geographical atrophy secondary to AMD, and it was concluded that retinal sensitivity changes are related to integrity of the photoreceptor ISel band. ${ }^{55}$
Several retrospective studies have evaluated the SDOCT changes in patients who were treated for neovascular AMD ${ }^{56-58}$ After being successfully treated with photodynamic therapy (PDT), it was found that eyes with a continuous or discontinuous photoreceptor ISel band beneath the fovea were associated with better final BCVA than eyes without an ISel band. Visual outcome was also found to be significantly better in eyes with PCV. At final visit, it was found that $86.7 \%$ of eyes with typical AMD had no photoreceptor ISel band, whereas $36.1 \%$ of eyes with PCV had no photoreceptor ISel band beneath the fovea. ${ }^{56}$ Other studies have evaluated the correlation between the outer retinal structural changes on SD-OCT and treatment outcome in patients treated with intravitreal antivascular endothelium growth factor (anti-VEGF) therapy..$^{57,58}$ In a study on patients with neovascular AMD treated with intravitreal bevacizumab, univariate analysis showed that pretreatment damage to ELM was associated with poor visual outcome. On multivariate analysis, pretreatment BCVA and integrity of the ELM and photoreceptor ISel band were found to be
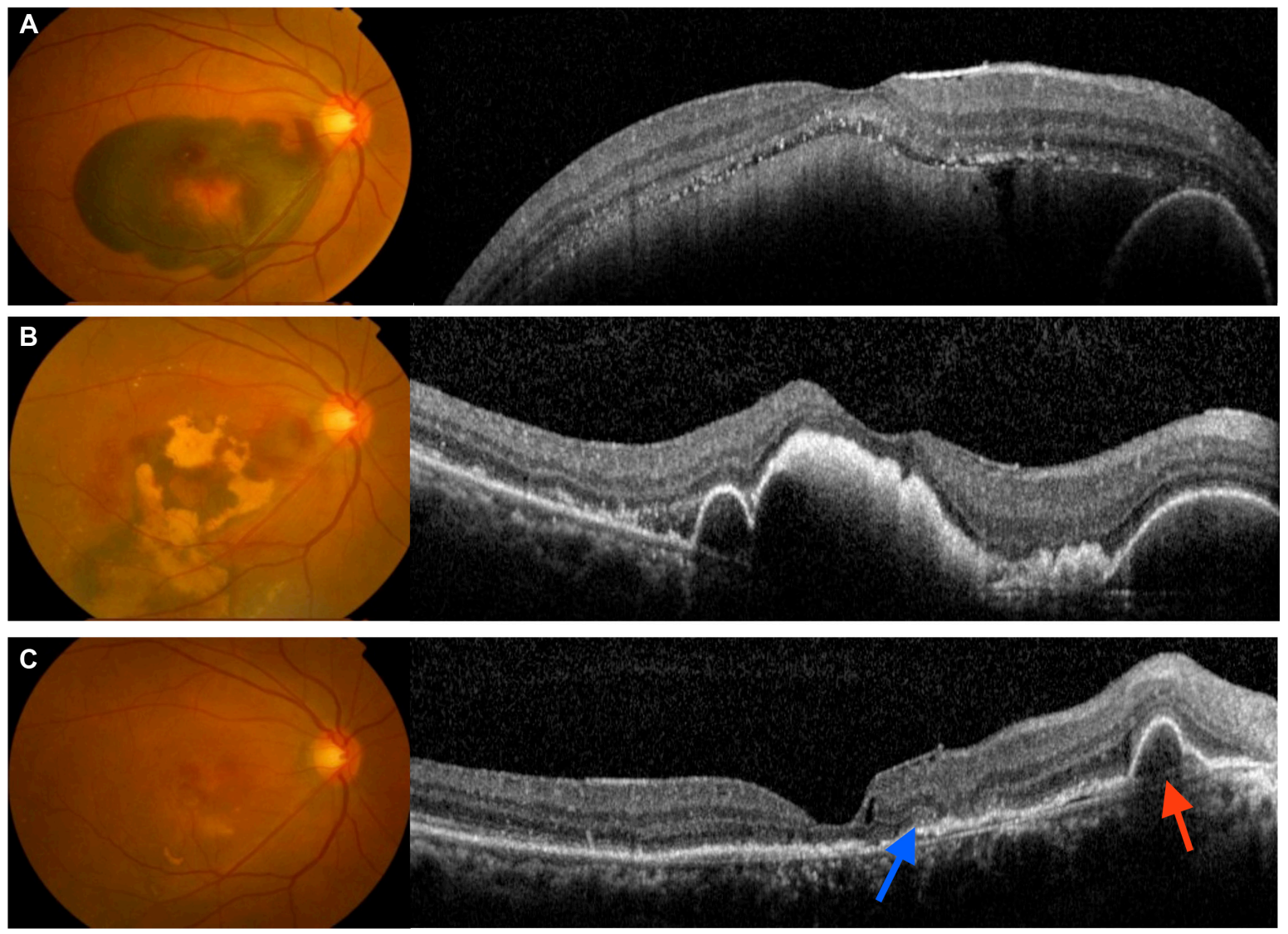

Figure 6 Submacular hemorrhage in neovascular AMD.

Notes: (A) large subretinal hemorrhage; (B) partial resolution of the subretinal hemorrhage with hemorrhagic pigment epithelium detachment and the ISel band could not be discerned; (C) restoration of the photoreceptor ISel band (blue arrow) with residual pigment epithelium detachment (red arrow).

Abbreviations: AMD, age-related macular degeneration; ISel, inner segment ellipsoid. 
important predictors of visual outcome in $37 \%$ of cases. ${ }^{55}$ Prognostic factors for good visual outcome following 3 monthly injections of intravitreal bevacizumab in patients with neovascular AMD included integrity of the photoreceptor ISel band and ELM, but baseline visual acuity had the best predictive value. ${ }^{58}$

In nonexudative $\mathrm{AMD}$, disruption of the photoreceptor ISel band may be associated with progression of drusen. ${ }^{59}$ Defects in the ISel band on SD-OCT and reduction in cone density using adaptive optics imaging have been described in eyes with subretinal drusenoid deposits. ${ }^{60}$ As these subretinal drusenoid deposits regress, loss of the ISel band can be observed, and this was shown to be associated with significant visual loss. ${ }^{60,61}$

\section{Myopic choroidal neovascularization hemorrhage}

The development of CNV is one of the most vision-threatening complications associated with pathologic myopia. Progression of myopic maculopathy occurs in a substantial proportion of patients resulting in significant visual loss. In the past decade, PDT with verteporfin has been used for treating myopic CNV. However, the long-term outcome of PDT is not favorable, as patients generally had no improvement in visual acuity following treatment. The short-term efficacy of intravitreal bevacizumab and ranibizumab has been demonstrated by various studies in treating myopic CNV.${ }^{62-79}$ Most of the studies have demonstrated significant mean visual improvement after anti-VEGF therapy, and the beneficial effects were maintained at 12 months. Several recent studies have also reported the long-term visual outcomes up to 2 years following intravitreal bevacizumab for myopic CNV.$^{68,80,81}$ As compared with the short-term results, these longer term results were more variable, as studies have reported that the initial visual gain might no longer be significant at 2 years. ${ }^{68,81}$ The microstructural changes and prognostic factors of final visual outcome in myopic subretinal hemorrhages without CNV were studied. The parameters investigated included the maximal hemorrhagic height, intraretinal hyperscattering signal (intraretinal hyperreflective sign) across the retina at 6 months and the integrity of the photoreceptor ISel band and the ELM. It was found that significant visual improvement and resolution of hemorrhages occurred in $92.3 \%$ of eyes by 6 months. The intraretinal hyperreflective sign in all eyes extended into the outer nuclear layer in five $(38.5 \%)$ eyes, to the ILM in four $(30.8 \%)$ eyes, and between the two layers in the remaining four $(30.8 \%)$ eyes. The location of the hyperreflective signs at 6 months coincided with the ruptured retinal layers at baseline in all eyes. Moreover, the photoreceptor ISel band and the ELM were found to be intact in $46.2 \%$ of the eyes. Significant association was found between BCVA and integrity of the photoreceptor ISel band and ELM. It was concluded that correlation exists between the photoreceptor function and the intraretinal hyperreflective sign, which was presumed to be due to scarring through the disrupted outer retina. ${ }^{82}$

\section{Diabetic macular edema}

Diabetic retinopathy can result in structural changes in retina and the extent of the structural changes have been shown to correlate with the severity of retinopathy. OCT has been extremely useful to demonstrate various structural changes in patients with diabetic macular edema (DME) (Figure 7). Currently, there is still no universally accepted classification of DME based on OCT. However, five common patterns have been described, including diffuse retinal edema, cystoid macular edema, serous retinal detachment, posterior hyaloidal traction, and tractional retinal detachment. ${ }^{83}$ Photoreceptor dysfunction may be a significant predictor of visual acuity in patients with diabetic retinopathy. Several retrospective observational studies were conducted to establish the correlation between photoreceptor ISel band and visual acuity in patients with DME. Maheshwary et $\mathrm{al}^{84}$ graded ISel disruption from grade $0-2$, with grade 0 being intact ISel, grade 1 being focal ISel disruption of $200 \mu \mathrm{m}$ or less, and grade 2 being ISel disruption of more than $200 \mu \mathrm{m}$. Grades from each patient's horizontal and vertical scan were added to yield a global disruption scale. They demonstrated that the percentage of photoreceptor ISel band disruption would be an important predictor of visual acuity among DME patients. ${ }^{84}$ Previous study has shown that the transverse length of the disrupted or absent ISel band has been related to visual impairment. ${ }^{85}$ Our recent study demonstrated that increased levels of serum

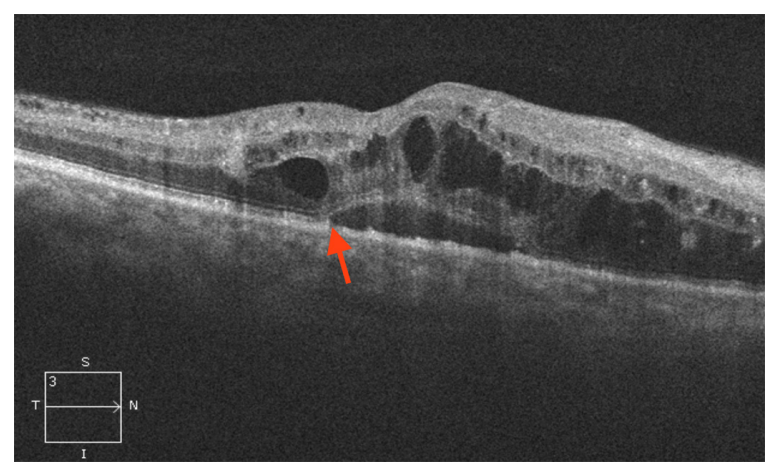

Figure 7 Diabetic macular edema showing disruption of both photoreceptor ISel band and ELM (red arrow), large cystic spaces, and serous detachment.

Abbreviations: ISel, inner segment ellipsoid; ELM, external limiting membrane; $\mathrm{S}$, superior; N, nasal; I, inferior; T, temporal. 
vascular endothelial growth factor and intercellular adhesion molecule-1 levels in diabetic retinopathy are associated with an increase in the severity of diabetic retinopathy and the grade of subfoveal ELM and ISel band disruption. Disruption of the photoreceptor ISel band and ELM was graded by SD-OCT as follows: grade 0 being no disruption of ELM and ISel band (Figure 8A), grade 1 being ELM disrupted with intact ISel band (Figure 8B), and grade 2 having both

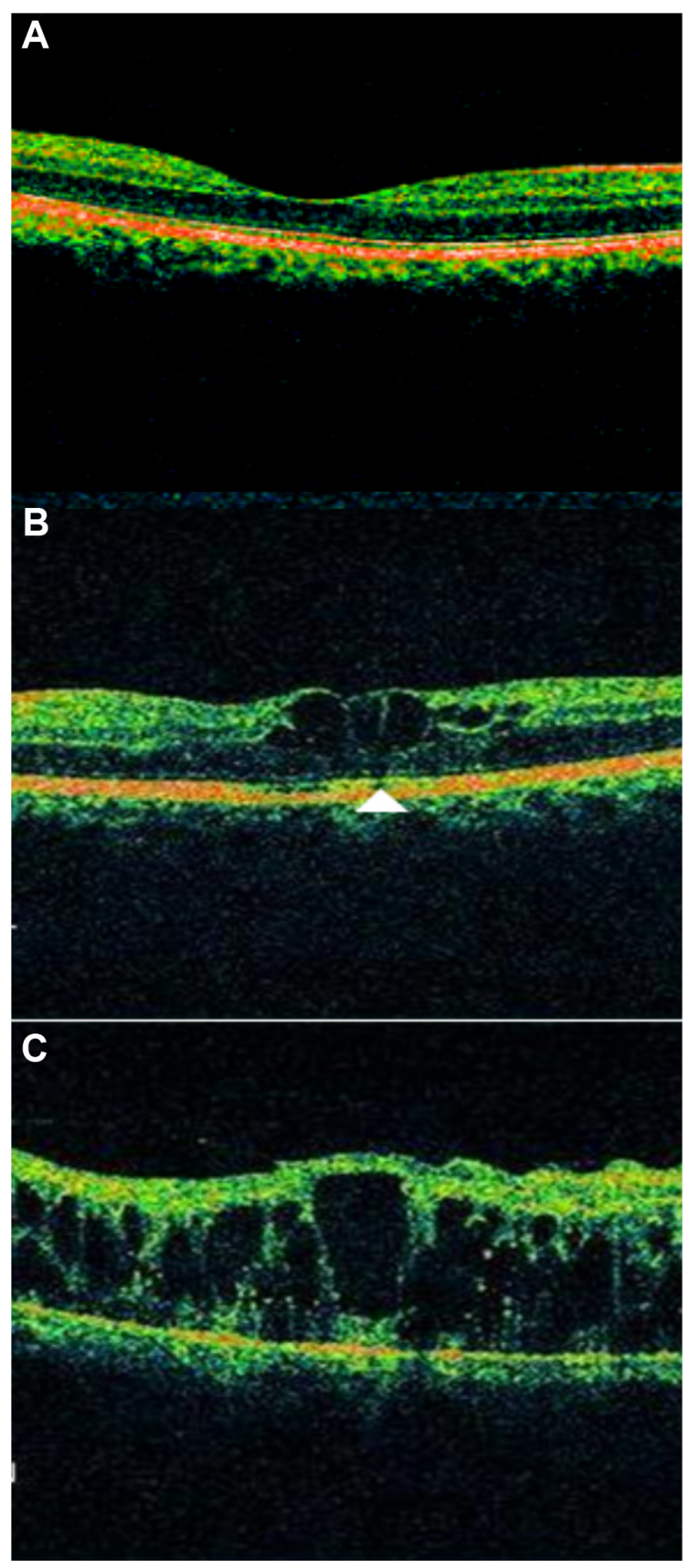

Figure 8 Spectral domain optical coherence tomography (SD-OCT) showing various grades of ELM and ISel band disruption.

Notes: (A) grade 0, no disruption of ELM and ISel; (B) grade I, ELM disrupted, ISel intact (arrow head); and (C) grade 2, both ELM and ISel disrupted.

Abbreviations: ISel, inner segment ellipsoid; ELM, external limiting membrane.
ELM and ISel band disrupted (Figure 8C). ${ }^{86}$ Disrupted ELM and photoreceptor ISel band on SD-OCT is associated with hyperreflective foci in the outer retina and visual outcomes in DME ${ }^{87}$ However, only a few interventional studies have been conducted in this area. A statistically significant association of intact ELM and ISel band in the fovea with BCVA was found in patients with resolved macular edema following pars plana vitrectomy with ILM removal for DME. ${ }^{88}$ In a retrospective interventional study, patients with DME who were successfully treated with intravitreal triamcinolone were included. Based on the restoration of the photoreceptor ISel band and ELM on SD-OCT at the final visit, eyes were divided into three groups: 1) group A with a completely visible ISel band and ELM, 2) group B with a disrupted ISel band and intact ELM, and 3) group C with a disrupted or loss of the ISel band and ELM. It was concluded that integrity of the photoreceptor ISel band correlated with final BCVA. Final BCVA of group A or group B was found to be better than group $\mathrm{C}$. They also concluded that shorter length of a disrupted photoreceptor ISel band and ELM was associated with better BCVA. However, on multivariate analysis, it was found that mean disrupted photoreceptor ISel band and ELM length showed greatest correlation with final BCVA compared with disrupted photoreceptor ISel band and ELM. They also found that pretreatment visual acuity and photoreceptor status helped in predicting the posttreatment recovery of the photoreceptor ISel band and subsequent visual recovery. ${ }^{89}$ Another study was performed on patients with DME who received intravitreal bevacizumab (IVB) injections. It was concluded that correlation between FAF with SD-OCT and visual acuity can help to predict restoration of photoreceptor ISel band integrity after treatment. ${ }^{90}$ SD-OCT findings in patients with DME who underwent vitrectomy demonstrated that median axial length was longer in those with a visible photoreceptor ISel band at 12 months after surgery, and longer axial length predicted better visual outcome after vitrectomy in DME. ${ }^{91}$

\section{Central retinal vein occlusion}

Several studies have been carried out to establish a correlation between integrity of the photoreceptor ISel band and final visual outcomes after resolution of macular edema associated with central retinal vein occlusion (CRVO) (Figure 9). ${ }^{92,93}$ The authors concluded that loss of foveal photoreceptor ISel band and inner retinal layers on SD-OCT significantly correlated with poorer visual acuity in CRVO patients. In another study, it was noted that factors indicating better visual acuity were shorter length of the photoreceptor ISel band and good visual acuity at initial presentation. ${ }^{94}$ 


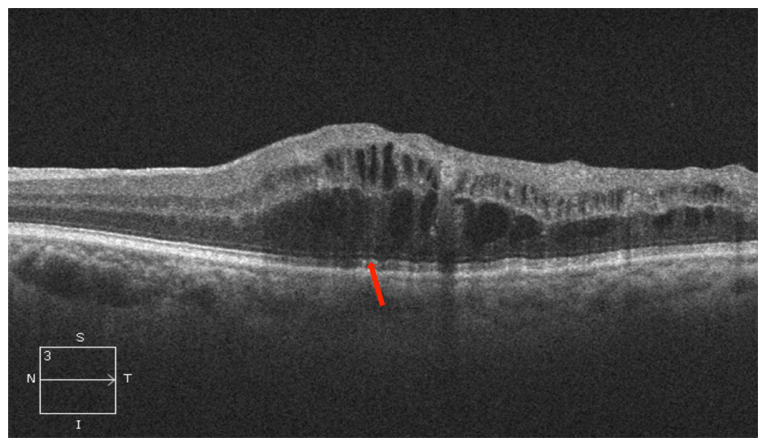

Figure 9 Central retinal vein occlusion associated with macular edema showing large cystic spaces associated with photoreceptor ISel band disruption (red arrow). Abbreviations: ISel, inner segment ellipsoid; S, superior; N, nasal; I, inferior; T, temporal.

\section{Macular telangiectasia}

Macular telangiectasia (MacTel) type 2 or juxtafoveal retinal telangiectasia is a bilateral disease of unknown cause with characteristic biomicroscopic and angiographic characteristics. Advances in imaging techniques, particularly using FAF imaging and SD-OCT, have led to remarkable progress in the understanding of MacTel (Figure 10). Reduction in macular pigment has been demonstrated in this condition, which is supported by the finding of loss of normal central attenuation of FAF. SD-OCT features in MacTel include loss or disruption of the photoreceptor ISel band and hyporeflective cavities at the level of the inner or outer retina and retinal atrophy in later stages. Integrity of the photoreceptor ISel band was found, which correlated with visual acuity. ${ }^{95}$ Studies have demonstrated that segmentation and en face imaging of the photoreceptor ISel band were found to correlate with visual function and aid in assessing disease severity in MacTel type 2.96-98

\section{Central serous chorioretinopathy}

Studies using SD-OCT have enhanced our understanding of the pathogenesis of central serous chorioretinopathy (CSC).

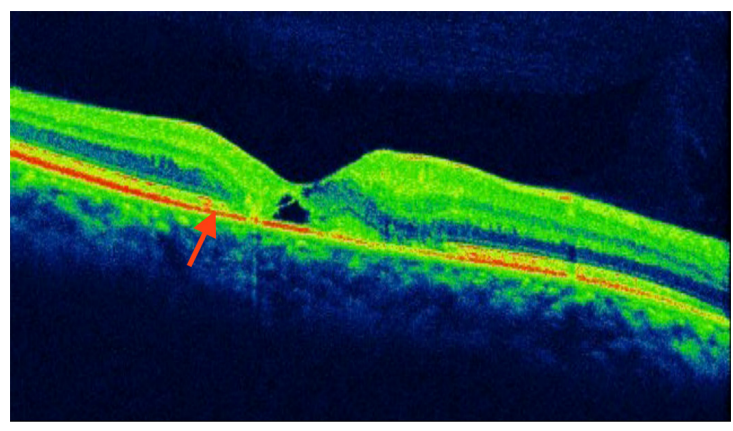

Figure 10 Macular telangiectasia showing juxtafoveal disruption of ISel band and ELM (red arrow).

Abbreviations: ISel, inner segment ellipsoid; ELM, external limiting membrane.

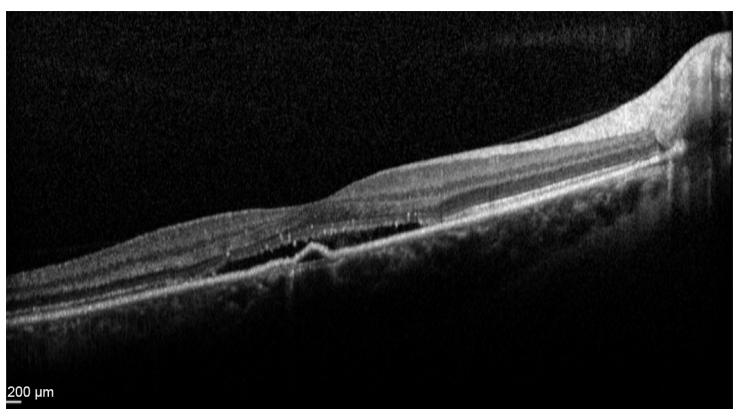

Figure II Acute central serous chorioretinopathy showing serous detachment with pigment epithelium detachment.

Note: Integrity of ISel band and ELM could not be discerned.

Abbreviations: ISel, inner segment ellipsoid; ELM, external limiting membrane.

A number of studies have been performed using SD-OCT to identify the retinal morphological changes in CSC (Figures 11 and 12). Visual loss in CSC is now believed to result from photoreceptor loss. SD-OCT has demonstrated disruption of the ISel band and loss of the COST line, presence of subretinal and intraretinal bright spots, decreased outer nuclear layer thickness and in chronic cases, posterior cystoid retinal degeneration. ${ }^{99-102}$ A prospective observational study noted that photoreceptor ISel band was missing before neurosensory retinal reattachment but later became present after reattachment. ${ }^{102}$ Visualization of the 3-D relationship between the ELM and each photoreceptor layer before and after macular reattachment have enhanced our understanding of the anatomical and visual changes due to CSC. In a similar study, ${ }^{103}$ correlation of the photoreceptor ISel band with prediction of visual recovery after macular reattachment was established. In another study, the photoreceptor ISel band was not visible in eyes with serous retinal detachment, but became visible after its resolution. ${ }^{104}$ Several studies have also evaluated the OCT changes in patients with chronic

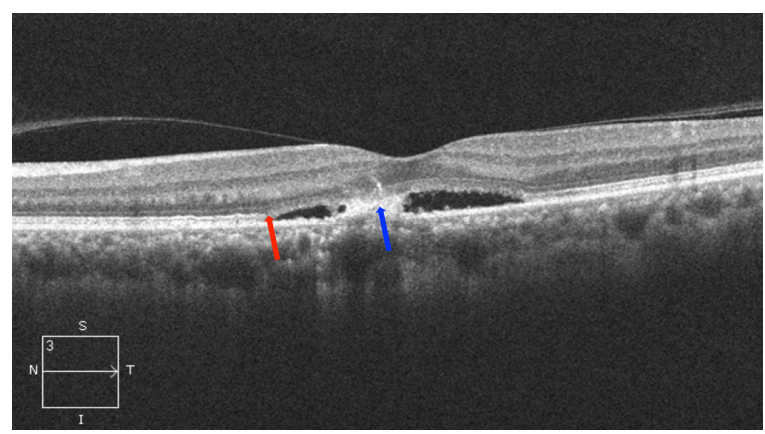

Figure 12 Chronic central serous chorioretinopathy showing disruption of ISel band and ELM (red arrow), and serous detachment and fibrin collection (blue arrow).

Note: Proliferating retinal pigment epithelial cells can also be seen.

Abbreviations: ISel, inner segment ellipsoid; ELM, external limiting membrane; $\mathrm{S}$, superior; N, nasal; I, inferior; T, temporal. 
CSC treated by PDT. Significant increase in macular sensitivity that correlated with recovery of photoreceptor ISel bands and COST lines was seen 6 and 12 months after halfdose verteporfin PDT. ${ }^{105}$ In another retrospective study on patients treated with standard PDT, it was concluded that morphological and functional changes correlated neither with the treatment nor with the progression of disease. ${ }^{106}$ Outer nuclear layer thickness reduction and discontinuity of the photoreceptor ISel band correlated with poor visual recovery in resolved CSC eyes following treatment with intravitreal ranibizumab. ${ }^{107}$ Various OCT parameters were evaluated to assess the visual function of eyes with resolved CSC. It was concluded that a disrupted photoreceptor ISel band was associated with worse BCVA. ${ }^{108}$ For accurate assessment of visual function, various parameters showing retinal status should be combined and interpreted together. ${ }^{109}$ Integrity of the ISel band has also correlated with functional assessment evaluated by microperimetry. ${ }^{110,111}$

\section{Retinitis pigmentosa}

A significant correlation between photoreceptor ISel band and visual acuity has been identified in patients with retinitis pigmentosa (RP). ${ }^{112}$ Assessment of the integrity of the photoreceptor ISel band was essential while monitoring cystoid macular edema associated with RP. ${ }^{113}$ Although the macular volume and length of the photoreceptor ISel band were shown to correlate weakly with the amplitude of focal macular electroretinography, a preserved macular morphology did not necessarily guarantee normal-amplitude focal macular electroretinography in RP patients. ${ }^{114}$ A few studies have attempted to correlate the FAF findings with the retinal structural changes in RP. According to one such study, FAF appeared to depict the integrity of the photoreceptor ISel band. ${ }^{115}$ Residual photoreceptor ISel band could be found inside the hyperautofluorescent ring, which correlated with visual outcome. ${ }^{116}$ During RP progression, the progressive constriction of the FAF ring correlated with the length of the photoreceptor ISel band. ${ }^{117}$ Presence of a photoreceptor ISel band can be considered to be an objective sign in predicting visual recovery after retinal prosthesis implantation. ${ }^{118}$ Eyes with cone-rod dystrophy (CRD) have significantly different features on SD-OCT compared with eyes with RP. Loss of the ISel band has been demonstrated in a significant proportion of patients with CRD or RP. However absence of an interdigitation zone and foveal cavitation have been shown to be more consistently seen in CRD. ${ }^{119,120}$ Similarly in eyes with achromatopsia, foveal ellipsoid zone disruption and foveal hypoplasia have been described on SD-OCT. ${ }^{121}$ An SD-OCT based staging system has been proposed recently to facilitate classification of this condition. ${ }^{121}$ In stage 1 , intact outer retina is seen; stage 2 had ISel band disruption; stage 3 showed presence of an optically empty space; stage 4 showed optically empty space with partial retinal pigment epithelium disruption; and stage 5 had complete retinal pigment epithelium disruption and/or loss of the outer nuclear layer. ${ }^{122}$

\section{Stargardt's disease}

SD-OCT of patients with reduced vision due to Stargardt's disease typically demonstrate disruption of the ISel band (Figure 13). Gomes et al concluded that there was a correlation between findings of SD-OCT and FAF in Stargardt's disease. They also suggested that structural changes affecting integrity of photoreceptors as seen in SD-OCT may occur earlier than changes in retinal pigment epithelium as detected on FAF. ${ }^{123}$ The extent of the photoreceptor ISel band preservation showed a correlation with the extension of foveal abnormalities assessed by FAF imaging. ${ }^{124}$ After mutation analysis, association of G1961E mutation of the $A B C A 4$ gene with milder Stargardt's disease phenotype was established, as patients with this mutation did not have extensive loss of photoreceptor ISel band. ${ }^{125}$

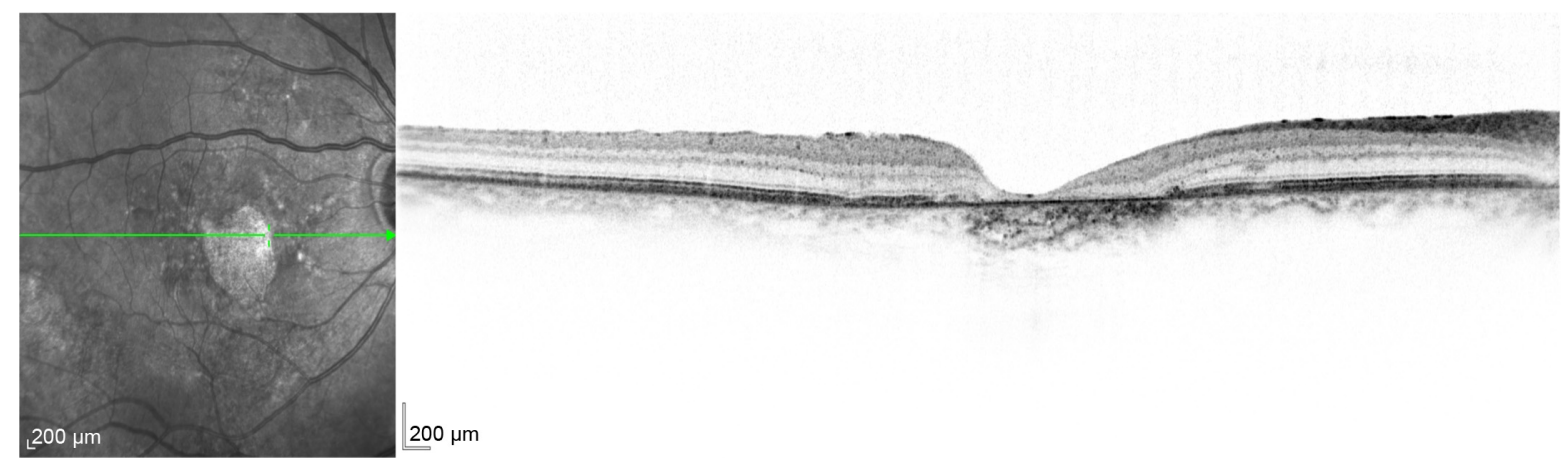

Figure 13 Stargardt's disease showing disruption of ISel band, increased foveal pit with hyperreflectivities at the level of retinal pigment epithelium, suggestive of flecks. Abbreviation: ISel, inner segment ellipsoid. 


\section{Occult macular dystrophy}

Occult macular dystrophy is a rare disorder which presents as bilaterally decreased visual acuity and occurs in the absence of any significant ophthalmoscopic findings. A study was conducted on five patients with markedly decreased visual acuity, and no abnormality was found on full field electroretinogram, fundus photography and FA. However, multifocal electroretinogram was abnormal. SD-OCT was conducted, and all patients showed decreased bowing, and few patients showed disruption of the photoreceptor ISel band. ${ }^{126}$ The findings of SD OCT were helpful in determining the pathophysiology and diagnostic criteria of occult macular dystrophy. ${ }^{126,127}$

\section{Acute zonal occult outer retinopathy complex}

Optical coherence tomography has demonstrated disruption of ISel in various chorioretinal inflammatory diseases including acute zonal occult outer retinopathy (AZOOR), multiple evanescent white dot syndrome (MEWDS), multifocal choroiditis and panuveitis, punctate inner choroidopathy (Figure 14), acute macular choroidopathy, and acute idiopathic blind spot enlargement syndrome. AZOOR is typified by acute loss of one or more zones of outer retinal function associated with photopsia, visual field defects, and minimal changes on fundus. Multifocal electroretinogram showed decreased response from the blind spots and other visual field defects with corresponding loss or disruption of the photoreceptor ISel band on SD-OCT. Hangai reported reflective focal lesions within the outer photoreceptor layer and disrupted ISel band, which corresponded to the hypofluorescent areas in the late phase of indocyanine green angiography in MEWDS. ${ }^{128}$ In addition, thinning of the outer nuclear layer has also been described. ${ }^{129}$ In several

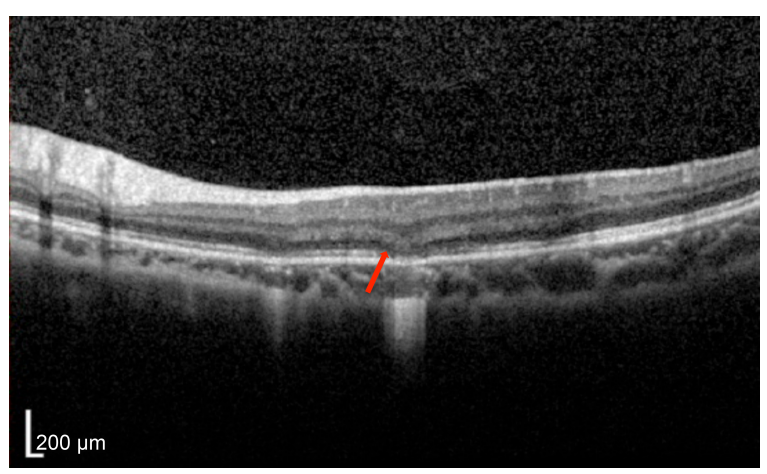

Figure I 4 Punctate inner choroidopathy showing disruption of photoreceptor ISel band and ELM (red arrow).

Abbreviations: ISel, inner segment ellipsoid; ELM, external limiting membrane. studies conducted to establish correlation between visual field defects and SD-OCT findings, it was found that disruption of photoreceptor ISel band correlated with blind spot enlargement. ${ }^{130}$ Photoreceptor ISel band disruption was found on SD-OCT in all patients with MEWDS as evidenced by a few studies. ${ }^{131-133}$

\section{Solar retinopathy}

Solar retinopathy can develop following exposure to arc welding, viewing of a solar eclipse, or direct sun gazing in religious ritual participants, military personnel, people with mental disturbances, and sunbathers (Figure 15). Damage to the inner and outer segment of the photoreceptors in patients with both acute and chronic presentations without involvement of the RPE was demonstrated in patients with solar retinopathy on SD-OCT. ${ }^{134} \mathrm{RPE}$ defects were more prominent in the acute exposure group, and photoreceptor defects were found more often in the chronic exposure group. While this was significant, RPE defects were still observed in the chronic exposure group, and photoreceptor defects were also seen in the acute exposure group. It was concluded that solar retinopathy mainly involved the inner photoreceptor segment. The authors of this study further added that visual loss in solar retinopathy can occur from chronic photoreceptor changes in the inner photoreceptor layer or at the junction of the inner and outer photoreceptor layers. A large fragment of hyporeflectance at the level of the outer and inner photoreceptor layer with an abrupt disruption of the photoreceptor ISel band was found to be correlated with decreased visual acuity. ${ }^{135}$ A retrospective observational study demonstrated defects in photoreceptor ISel bands with foveal atrophy in all patients on SD-OCT, whereas FA identified classic window defects in only a few eyes. ${ }^{136}$ In addition to distinct defects in the photoreceptor ISel band and photoreceptor outer segments, bilateral foveal thinning was demonstrated on high-speed ultra high definition OCT in a 34-year-old

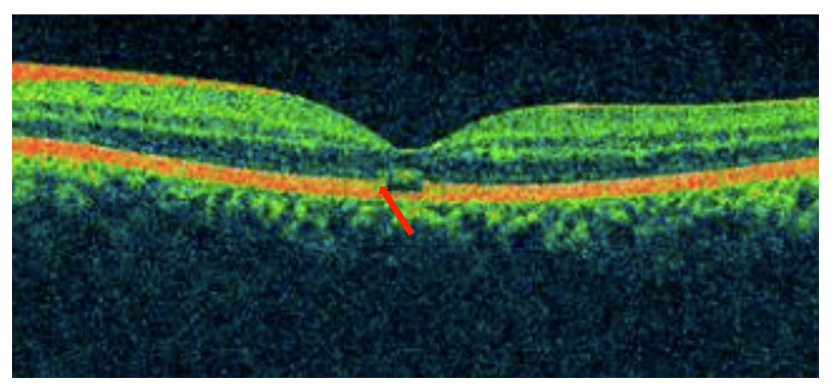

Figure 15 Solar retinopathy showing disruption of photoreceptor ISel band with intact ELM (red arrow).

Abbreviations: ISel, inner segment ellipsoid; ELM, external limiting membrane. 
man with chronic solar retinopathy. ${ }^{137}$ An observational case series demonstrated abnormally high reflectivity in the outer foveal retina as disruption of the photoreceptor ISel band and full thickness involvement of the photoreceptor layer was associated with poor visual acuity. ${ }^{138}$

\section{Uveitis}

Various retinal complications can occur in patients with uveitis and OCT has documented changes such as CNV, macular edema, and ISel band disruption in patients with uveitis (Figure 16). In a study, two morphological patterns were observed on SD-OCT performed on patients with uveitis macular edema: cystoid macular edema in $69 \%$ of eyes and diffuse macular edema in $31 \%$. It was concluded that BCVA negatively correlated with cystoid pattern and foveal thickening. In addition, a disrupted photoreceptor ISel band was also associated with poor visual acuity. ${ }^{139}$ A disrupted photoreceptor ISel band was associated with poor visual outcome in another study of patients with ERM secondary to uveitis. ${ }^{140}$

\section{Sympathetic ophthalmia}

Gupta et al conducted a study to investigate the photoreceptor changes occurring in the acute phase of sympathetic ophthalmia. ${ }^{141}$ Six consecutive patients with sympathetic ophthalmia underwent FA and SD-OCT assessments at presentation. Patients received intravenous methylprednisolone followed by oral corticosteroids and serial OCT examinations were performed 48 hours after the initiation of treatment and 1, 2, and 12 weeks later. Outer retinal segment showed serous retinal detachment and disruption to the continuity of the two inner hyperreflective bands in all the eyes and elongation of photoreceptors could be seen in four eyes. However, after 4 weeks, there was resolution of serous

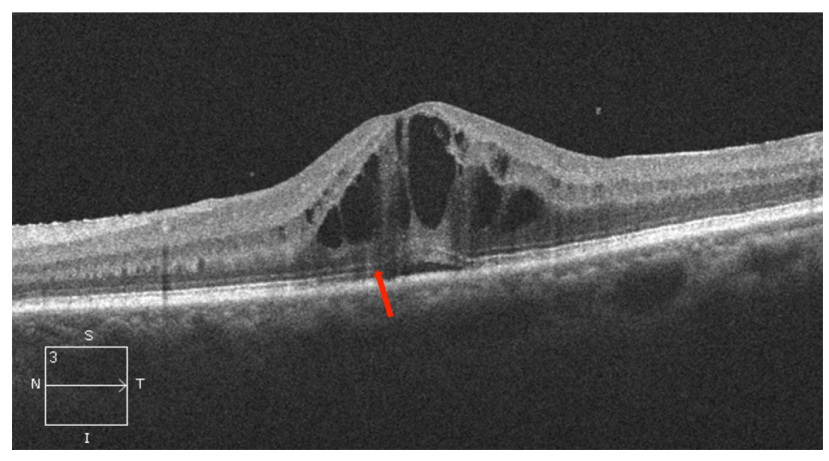

Figure 16 Uveitis associated with macular edema showing discontinuous photoreceptor ISel band and ELM (red arrow), and cystic spaces with serous detachment.

Abbreviations: ISel, inner segment ellipsoid; ELM, external limiting membrane; $\mathrm{S}$, superior; N, nasal; I, inferior; T, temporal. detachment and restoration of the photoreceptor ISel band. This study supported the hypothesis that prompt and aggressive anti-inflammatory therapy is capable of reversing the photoreceptor changes in sympathetic ophthalmia.

\section{Vogt-Koyanagi-Harada disease}

Vogt-Koyanagi-Harada (VKH) disease commonly occurs in pigmented individuals and can result in multifocal exudative retinal detachment during the acute phase. OCT is useful in documenting the changes in exudative retinal detachment following treatment for VKH disease (Figure 17A, B, and C). Ishihara et al conducted a study to evaluate membranous structures seen on SD-OCT in patients with acute VKH disease. ${ }^{142}$ It was noted that the membranous structure appeared to include a highly reflective line that seemed to be in conjunction with the photoreceptor ISel band in attached retina. Intraretinal split was seen to overlie the abnormal photoreceptor ISel band in the vicinity of cystoid spaces involving the fovea in nine $(45 \%)$ of the 20 eyes. During the course of corticosteroid therapy, the membranous structure changed to a granular structure. The authors hypothesized that inflammatory products like fibrin bound with the membranous form of the outer segment and corticosteroid therapy causes its dissolution, thereby changing the membranous structure into a granular structure.

\section{Behcet's disease}

A study by Unoki et al evaluated patients with remitting ocular Behcet's disease, and the correlation between SDOCT findings and BCVA was evaluated. Foveal thickness and integrity of the ELM and the photoreceptor ISel band on SD-OCT were analyzed, and it was found that visual acuity correlated with integrity of the photoreceptor ISel band and not with the ELM. ${ }^{143}$

\section{Choroidal hemangioma}

Choroidal hemangioma is a benign vascular tumor arising in the choroidal and can result in visual loss due to exudation (Figure 18). A retrospective review of 14 eyes with symptomatic circumscribed choroidal hemangioma that was treated with PDT was evaluated using time-domain OCT. ${ }^{144}$ It was found that a preserved photoreceptor ISel band was associated with good visual acuity after PDT.

\section{Drug-related maculopathies}

Several studies were conducted to correlate visual field defects with SD-OCT findings in patients who developed retinal toxicity after treatment with hydroxychloroquine 


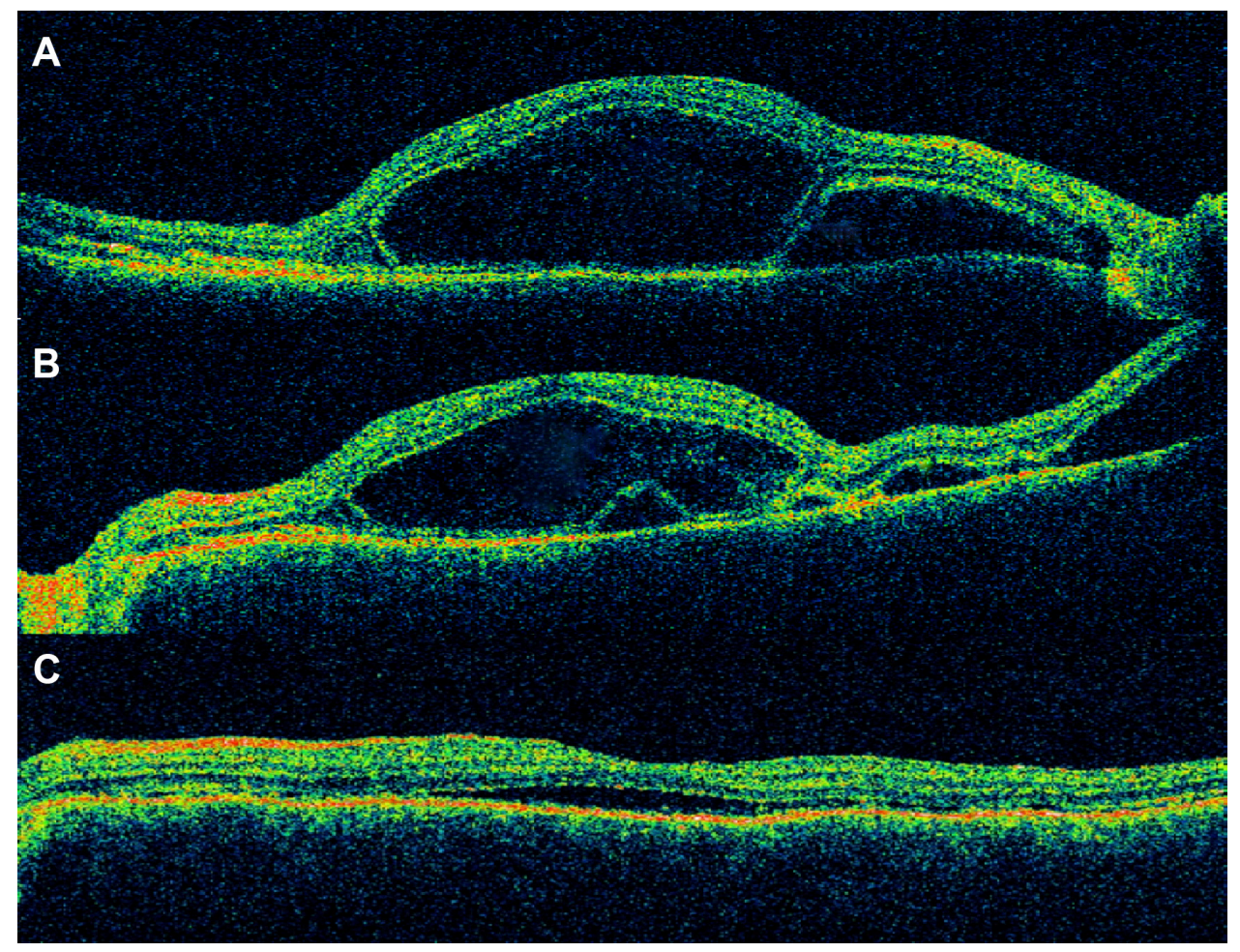

Figure $17 \mathrm{VKH}$ disease after treatment with intravenous methylprednisolone showing (A) subretinal fluid, (B) resolving subretinal fluid, and (C) residual subretinal fluid. Note: The integrity of ISel band and ELM has not been restored yet.

Abbreviations: VKH, Vogt-Koyanagi-Harada; ISel, inner segment ellipsoid; ELM, external limiting membrane.

or chloroquine. ${ }^{145-147}$ It was found that structural changes in the photoreceptor ISel band correlated topographically with field defects seen on Humphrey visual field 10-2. Moreover, SD-OCT may be helpful in determining subclinical abnormalities. ${ }^{145}$ It has been shown that photoreceptor ISel band changes correlated with changes on multifocal electroretinography. ${ }^{146,147}$ Findings of FAF imaging were consistent with disruptions in photoreceptor ISel band in patients who developed hydroxychloroquine maculopathy. ${ }^{148}$ To this effect, SD-OCT was listed as one of the recommended screening procedures for early detection of hydroxychloroquine toxicity according to the revised American Academy of Ophthalmology guideline. ${ }^{149}$

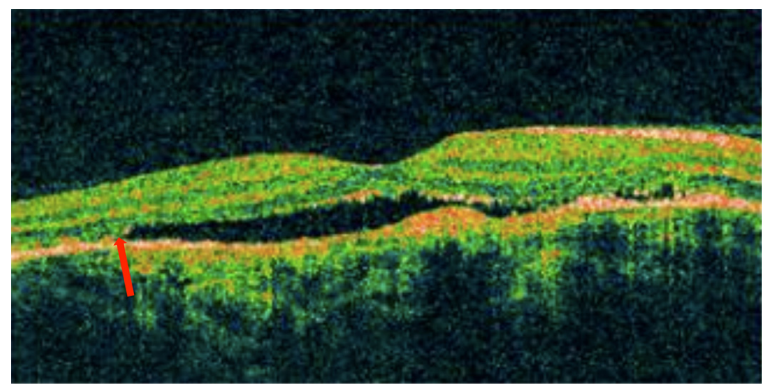

Figure 18 Choroidal hemangioma showing disruption of photoreceptor ISel band, ELM (red arrow) and serous detachment.

Abbreviations: ISel, inner segment ellipsoid; ELM, external limiting membrane.

\section{Conclusion}

Based on this review, we conclude that photoreceptor dysfunction may be a significant predictor of visual acuity in a wide spectrum of surgical and medical retinal diseases. Both preoperative and postoperative disruption of ISel band has been found to correlate with BCVA. In addition to the ISel band, various other OCT parameters including ELM and COST line can be evaluated to determine the prognosis of various retinal diseases.

\section{Disclosure}

The authors have no conflicts of interest in this work.

\section{References}

1. Chang LK, Koizumi H, Spaide RF. Disruption of the photoreceptor inner segment-outer segment junction in eyes with macular holes. Retina. 2008;28(7):969-975.

2. Spaide RF, Koizumi H, Freund KB. Photoreceptor outer segment abnormalities as a cause of blind spot enlargement in acute zonal occult outer retinopathy-complex diseases. Am J Ophthalmol. 2008;146(1): $111-120$

3. Wang NK, Chou CL, Lima LH, et al. Fundus autofluorescence in cone dystrophy. Doc Ophthalmol. 2009;119(2):141-144.

4. Oh J, Smiddy WE, Flynn HW Jr, Gregori G, Lujan B. Photoreceptor inner/outer segment defect imaging by spectral domain OCT and visual prognosis after macular hole surgery. Invest Ophthalmol Vis Sci. 2010;51(3):1651-1658. 
5. Inoue M, Morita S, Watanabe Y, et al. Inner segment/outer segment junction assessed by spectral-domain optical coherence tomography in patients with idiopathic epiretinal membrane. Am J Ophthalmol. 2010; 150(6):834-839.

6. Hood DC, Lazow MA, Locke KG, Greenstein VC, Birch DG. The transition zone between healthy and diseased retina in patients with retinitis pigmentosa. Invest Ophthalmol Vis Sci. 2011;52(1):101-108.

7. Kitaguchi Y, Kusaka S, Yamaguchi T, Mihashi T, Fujikado T. Detection of photoreceptor disruption by adaptive optics fundus imaging and Fourier-domain optical coherence tomography in eyes with occult macular dystrophy. Clin Ophthalmol. 2011;5:345-351.

8. Drexler W, Sattmann H, Hermann B, et al. Enhanced visualization of macular pathology with the use of ultrahigh-resolution optical coherence tomography. Arch Ophthalmol. 2003;121(5):695-706.

9. Ko TH, Fujimoto JG, Duker JS, et al. Comparison of ultrahigh- and standard-resolution optical coherence tomography for imaging macular hole pathology and repair. Ophthalmology. 2004;111(11):2033-2043.

10. Srinivasan VJ, Ko TH, Wojtkowski M, et al. Noninvasive volumetric imaging and morphometry of the rodent retina with high-speed, ultrahigh-resolution optical coherence tomography. Invest Ophthalmol Vis Sci. 2006;47(12):5522-5528.

11. Srinivasan VJ, Monson BK, Wojtkowski M, et al. Characterization of outer retinal morphology with high-speed, ultrahigh-resolution optical coherence tomography. Invest Ophthalmol Vis Sci. 2008; 49(4):1571-1579.

12. Oishi A, Hata M, Shimozono M, Mandai M, Nishida A, Kurimoto Y. The significance of external limiting membrane status for visual acuity in age-related macular degeneration. Am J Ophthalmol. 2010;150(1) 27-32.e1.

13. Zawadzki RJ, Jones SM, Olivier SS, et al. Adaptive-optics optical coherence tomography for high-resolution and high-speed 3D retinal in vivo imaging. Opt Express. 2005;13(21):8532-8546.

14. Puche N, Querques G, Benhamou N, et al. High-resolution spectral domain optical coherence tomography features in adult onset foveomacular vitelliform dystrophy. Br J Ophthalmol. 2010;94(9):1190-1196.

15. Spaide RF, Curcio CA. Anatomical correlates to the bands seen in the outer retina by optical coherence tomography: literature review and model. Retina. 2011;31(8):1609-1619.

16. Anger EM, Unterhuber A, Hermann B, et al. Ultrahigh resolution optical coherence tomography of the monkey fovea. Identification of retinal sublayers by correlation with semithin histology sections. Exp Eye Res. 2004; 78(6):1117-1125.

17. Spaide RF. Questioning optical coherence tomography. Ophthalmology. 2012;119(11):2203-2204.e1.

18. Krebs W, Krebs I. Primate Retina and Choroid: Atlas of Fine Structure in Man and Monkey. New York, NY: Springer-Verlag; 1991.

19. Hogan JA, Alvarado MJ, Weddell JE. Histology of the Human Eye: An Atlas and Textbook. Philadelphia, PA: WB Saunders; 1971.

20. Fernandez EJ, Hermann B, Povazay B, et al. Ultrahigh resolution optical coherence tomography and pancorrection for cellular imaging of the living human retina. Opt Express. 2008;16(15):11083-11094.

21. Lu RW, Curcio CA, Zhang Y, et al. Investigation of the hyper-reflective inner/outer segment band in optical coherence tomography of living frog retina. J Biomed Opt. 2012;17(6):060504.

22. Fernandez EJ, Povazay B, Hermann B, et al. Three-dimensional adaptive optics ultrahigh-resolution optical coherence tomography using a liquid crystal spatial light modulator. Vision Res. 2005;45(28):3432-3444.

23. Zhang Y, Cense B, Rha J, et al. High-speed volumetric imaging of cone photoreceptors with adaptive optics spectral-domain optical coherence tomography. Opt Express. 2006;14(10):4380-4394.

24. Sano M, Shimoda Y, Hashimoto H, Kishi S. Restored photoreceptor outer segment and visual recovery after macular hole closure. Am J Ophthalmol. 2009;147(2):313-318.e1.

25. Baba T, Yamamoto S, Arai M, et al. Correlation of visual recovery and presence of photoreceptor inner/outer segment junction in optical coherence images after successful macular hole repair. Retina. 2008;28(3): 453-458.
26. Wakabayashi T, Fujiwara M, Sakaguchi H, Kusaka S, Oshima Y. Foveal microstructure and visual acuity in surgically closed macular holes: spectral-domain optical coherence tomographic analysis. Ophthalmology. 2010;117(9):1815-1824.

27. Bottoni F, De Angelis S, Luccarelli S, Cigada M, Staurenghi G. The dynamic healing process of idiopathic macular holes after surgical repair: a spectral-domain optical coherence tomography study. Invest Ophthalmol Vis Sci. 2011;52(7):4439-4446.

28. Theodossiadis PG, Grigoropoulos VG, Theodossiadis GP. The significance of the external limiting membrane in the recovery of photoreceptor layer after successful macular hole closure: a study by spectral domain optical coherence tomography. Ophthalmologica. 2011; 225(3):176-184.

29. Shimozono M, Oishi A, Hata M, Kurimoto Y. Restoration of the photoreceptor outer segment and visual outcomes after macular hole closure: spectral-domain optical coherence tomography analysis. Graefes Arch Clin Exp Ophthalmol. 2011;249(10):1469-1476.

30. Ruiz-Moreno JM, Lugo F, Montero JA, Pinero DP. Restoration of macular structure as the determining factor for macular hole surgery outcome. Graefes Arch Clin Exp Ophthalmol. 2012;250(10):1409-1414.

31. Itoh Y, Inoue M, Rii T, Hiraoka T, Hirakata A. Significant correlation between visual acuity and recovery of foveal cone microstructures after macular hole surgery. Am J Ophthalmol. 2012;153(1):111-119.e1.

32. Mester V, Kuhn F. Internal limiting membrane removal in the management of full-thickness macular holes. Am J Ophthalmol. 2000;129(6): $769-777$.

33. Mitamura Y, Hirano K, Baba T, Yamamoto S. Correlation of visual recovery with presence of photoreceptor inner/outer segment junction in optical coherence images after epiretinal membrane surgery. $\mathrm{Br} J$ Ophthalmol. 2009;93(2):171-175.

34. Suh MH, Seo JM, Park KH, Yu HG. Associations between macular findings by optical coherence tomography and visual outcomes after epiretinal membrane removal. Am J Ophthalmol. 2009;147(3): 473-480.e3.

35. Oster SF, Mojana F, Brar M, Yuson RM, Cheng L, Freeman WR. Disruption of the photoreceptor inner segment/outer segment layer on spectral domain-optical coherence tomography is a predictor of poor visual acuity in patients with epiretinal membranes. Retina. 2010;30(5): 713-718.

36. Falkner-Radler CI, Glittenberg C, Hagen S, Benesch T, Binder S. Spectral-domain optical coherence tomography for monitoring epiretinal membrane surgery. Ophthalmology. 2010;117(4):798-805.

37. Theodossiadis PG, Theodossiadis GP, Charonis A, Emfietzoglou I, Grigoropoulos VG, Liarakos VS. The photoreceptor layer as a prognostic factor for visual acuity in the secondary epiretinal membrane after retinal detachment surgery: imaging analysis by spectral-domain optical coherence tomography. Am J Ophthalmol. 2011;151(6):973-980.

38. Brito PN, Gomes NL, Vieira MP, et al. Possible role for fundus autofluorescence as a predictive factor for visual acuity recovery after epiretinal membrane surgery. Retina. 2014;34(2):273-280.

39. Okamoto F, Sugiura Y, Okamoto Y, Hiraoka T, Oshika T. Associations between metamorphopsia and foveal microstructure in patients with epiretinal membrane. Invest Ophthalmol Vis Sci. 2012;53(11):6770-6775.

40. Shimozono M, Oishi A, Hata M, et al. The significance of cone outer segment tips as a prognostic factor in epiretinal membrane surgery. Am J Ophthalmol. 2012;153(4):698-704, 704.e1.

41. Watanabe K, Tsunoda K, Mizuno Y, Akiyama K, Noda T. Outer retinal morphology and visual function in patients with idiopathic epiretinal membrane. JAMA Ophthalmol. 2013;131(2):172-177.

42. Cobos E, Arias L, Ruiz-Moreno J, et al. Preoperative study of the inner segment/outer segment junction of photoreceptors by spectral-domain optical coherence tomography as a prognostic factor in patients with epiretinal membranes. Clin Ophthalmol. 2013;7:1467-1470.

43. Shiono A, Kogo J, Klose G, et al. Photoreceptor outer segment length: a prognostic factor for idiopathic epiretinal membrane surgery. Ophthalmology. 2013;120(4):788-794. 
44. Bae SH, Kim D, Park TK, Han JR, Kim H, Nam W. Preferential hyperacuity perimeter and prognostic factors for metamorphopsia after idiopathic epiretinal membrane surgery. Am J Ophthalmol. 2013; 155(1):109-117.e3.

45. Schocket LS, Witkin AJ, Fujimoto JG, et al. Ultrahigh-resolution optical coherence tomography in patients with decreased visual acuity after retinal detachment repair. Ophthalmology. 2006;113(4):666-672.

46. Wakabayashi T, Oshima Y, Fujimoto H, et al. Foveal microstructure and visual acuity after retinal detachment repair: imaging analysis by Fourier-domain optical coherence tomography. Ophthalmology. 2009; 116(3):519-528.

47. Cho M, Witmer MT, Favarone G, Chan RP, D'Amico DJ, Kiss S. Optical coherence tomography predicts visual outcome in macula-involving rhegmatogenous retinal detachment. Clin Ophthalmol. 2012;6:91-96.

48. Delolme MP, Dugas B, Nicot F, Muselier A, Bron AM, CreuzotGarcher C. Anatomical and functional macular changes after rhegmatogenous retinal detachment with macula off. Am J Ophthalmol. 2012; 153(1):128-136.

49. Joe SG, Kim YJ, Chae JB, et al. Structural recovery of the detached macula after retinal detachment repair as assessed by optical coherence tomography. Korean J Ophthalmol. 2013;27(3):178-185.

50. Akkoyun I, Yilmaz G. [Optical coherence tomography: anatomic and functional outcome after scleral buckling surgery in maculaoff rhegmatogenous retinal detachment]. Klin Monbl Augenheilkd. 2013;230(8):814-819. German.

51. Fujimoto S, Ikuno Y, Nishida K. Postoperative optical coherence tomographic appearance and relation to visual acuity after vitrectomy for myopic foveoschisis. Am J Ophthalmol. 2013;156(5):968-973.e1.

52. Saxena S, Manisha, Meyer CH. Three-dimensional spectral domain optical coherence tomography in $\mathrm{X}$ linked foveal retinoschisis. $B M J$ Case Rep. 2013;2013.

53. Sayanagi K, Ikuno Y, Soga K, Tano Y. Photoreceptor inner and outer segment defects in myopic foveoschisis. Am J Ophthalmol. 2008; 145(5):902-908

54. Gerendas BS, Kroisamer JS, Sulzbacher F, Schmidt-Erfurth U. Neovascular age-related macular degeneration. In: Midena E, editor. Microperimetry and Multimodal Retinal Imaging. Berlin, Heidelberg: Springer-Verlag Berlin Heidelberg; 2014

55. Pilotto E, Benetti E, Convento E, et al. Microperimetry, fundus autofluorescence, and retinal layer changes in progressing geographic atrophy. Can J Ophthalmol. 2013;48(5):386-393.

56. Hayashi H, Yamashiro K, Tsujikawa A, Ota M, Otani A, Yoshimura N. Association between foveal photoreceptor integrity and visual outcome in neovascular age-related macular degeneration. Am J Ophthalmol. 2009;148(1):83-89.e1.

57. Chhablani J, Kim JS, Freeman WR, Kozak I, Wang HY, Cheng L. Predictors of visual outcome in eyes with choroidal neovascularization secondary to age related macular degeneration treated with intravitreal bevacizumab monotherapy. Int J Ophthalmol. 2013;6(1):62-66.

58. Oishi A, Shimozono M, Mandai M, Hata M, Nishida A, Kurimoto Y. Recovery of photoreceptor outer segments after anti-VEGF therapy for age-related macular degeneration. Graefes Arch Clin Exp Ophthalmol. 2013;251(2):435-440.

59. Hartmann KI, Gomez ML, Bartsch DU, Schuster AK, Freeman WR. Effect of change in drusen evolution on photoreceptor inner segment/ outer segment junction. Retina. 2012;32(8):1492-1499.

60. Mrejen S, Sato T, Curcio CA, Spaide RF. Assessing the cone photoreceptor mosaic in eyes with pseudodrusen and soft Drusen in vivo using adaptive optics imaging. Ophthalmology. 2014;121(2):545-551.

61. Curcio CA, Messinger JD, Sloan KR, McGwin G, Medeiros NE, Spaide RF. Subretinal drusenoid deposits in non-neovascular agerelated macular degeneration: morphology, prevalence, topography, and biogenesis model. Retina. 2013;33(2):265-276.

62. Yamamoto I, Rogers AH, Reichel E, Yates PA, Duker JS. Intravitreal bevacizumab (Avastin) as treatment for subfoveal choroidal neovascularisation secondary to pathological myopia. Br J Ophthalmol. 2007; 91(2):157-160.
63. Sakaguchi H, Ikuno Y, Gomi F, et al. Intravitreal injection of bevacizumab for choroidal neovascularisation associated with pathological myopia. Br J Ophthalmol. 2007;91(2):161-165.

64. Chan WM, Lai TY, Liu DT, Lam DS. Intravitreal bevacizumab (Avastin) for myopic choroidal neovascularization: six-month results of a prospective pilot study. Ophthalmology. 2007;114(12):2190-2196.

65. Hernandez-Rojas ML, Quiroz-Mercado H, Dalma-Weiszhausz J, et al. Short-term effects of intravitreal bevacizumab for subfoveal choroidal neovascularization in pathologic myopia. Retina. 2007; 27(6):707-712.

66. Chan WM, Lai TY, Chan KP, et al. Changes in aqueous vascular endothelial growth factor and pigment epithelial-derived factor levels following intravitreal bevacizumab injections for choroidal neovascularization secondary to age-related macular degeneration or pathologic myopia. Retina. 2008;28(9):1308-1313.

67. Chan WM, Lai TY, Liu DT, Lam DS. Intravitreal bevacizumab (Avastin) for myopic choroidal neovascularisation: 1-year results of a prospective pilot study. Br J Ophthalmol. 2009;93(2):150-154.

68. Ikuno Y, Nagai Y, Matsuda S, et al. Two-year visual results for older Asian women treated with photodynamic therapy or bevacizumab for myopic choroidal neovascularization. Am J Ophthalmol. 2010;149(1): 140-146.

69. Ruiz-Moreno JM, Montero JA, Arias L, et al. Twelve-month outcome after one intravitreal injection of bevacizumab to treat myopic choroidal neovascularization. Retina. 2010;30(10):1609-1615.

70. Scupola A, Tiberti AC, Sasso P, et al. Macular functional changes evaluated with MP-1 microperimetry after intravitreal bevacizumab for subfoveal myopic choroidal neovascularization: one-year results. Retina. 2010;30(5):739-747.

71. Wakabayashi T, Ikuno Y, Gomi F. Different dosing of intravitreal bevacizumab for choroidal neovascularization because of pathologic myopia. Retina. 2011;31(5):880-886.

72. Gharbiya M, Giustolisi R, Allievi F, et al. Choroidal neovascularization in pathologic myopia: intravitreal ranibizumab versus bevacizumab a randomized controlled trial. Am J Ophthalmol. 2010;149(3): 458-464.e1.

73. Silva RM, Ruiz-Moreno JM, Nascimento J, et al. Short-term efficacy and safety of intravitreal ranibizumab for myopic choroidal neovascularization. Retina. 2008;28(8):1117-1123.

74. Lai TY, Chan WM, Liu DT, Lam DS. Intravitreal ranibizumab for the primary treatment of choroidal neovascularization secondary to pathologic myopia. Retina. 2009;29(6):750-756.

75. Mones JM, Amselem L, Serrano A, Garcia M, Hijano M. Intravitreal ranibizumab for choroidal neovascularization secondary to pathologic myopia: 12-month results. Eye (Lond). 2009;23(6):1275-1280; quiz 1281

76. Lalloum F, Souied EH, Bastuji-Garin S, et al. Intravitreal ranibizumab for choroidal neovascularization complicating pathologic myopia. Retina. 2010;30(3):399-406.

77. Silva RM, Ruiz-Moreno JM, Rosa P, et al. Intravitreal ranibizumab for myopic choroidal neovascularization: 12-month results. Retina. 2010;30(3):407-412.

78. Varano M, Tedeschi M, Oddone F, Perillo L, Coppe AM, Parravano M. Microperimetric retinal changes in myopic choroidal neovascularization treated with intravitreal ranibizumab. Retina. 2010;30(3):413-417.

79. Heier JS, Brown D, Ciulla T, et al. Ranibizumab for choroidal neovascularization secondary to causes other than age-related macular degeneration: a phase I clinical trial. Ophthalmology. 2011;118(1):111-118.

80. Gharbiya M, Allievi F, Conflitti S, et al. Intravitreal bevacizumab for treatment of myopic choroidal neovascularization: the second year of a prospective study. Clin Ter. 2010;161(3):e87-e93.

81. Ruiz-Moreno JM, Montero JA. Intravitreal bevacizumab to treat myopic choroidal neovascularization: 2-year outcome. Graefes Arch Clin Exp Ophthalmol. 2010;248(7):937-941.

82. Asai T, Ikuno Y, Nishida K. Macular microstructures and prognostic factors in myopic subretinal hemorrhages. Invest Ophthalmol Vis Sci. 2014;55(1):226-232. 
83. Kim BY, Smith SD, Kaiser PK. Optical coherence tomographic patterns of diabetic macular edema. Am J Ophthalmol. 2006;142(3):405-412.

84. Maheshwary AS, Oster SF, Yuson RM, Cheng L, Mojana F, Freeman WR. The association between percent disruption of the photoreceptor inner segment-outer segment junction and visual acuity in diabetic macular edema. Am J Ophthalmol. 2010;150(1):63-67.e1.

85. Saxena S, Jain A. Alterations in "in vivo" histology of retina in bilateral chronic central serous chorioretinopathy after intravitreal bevacizumab. J Ocul Biol Dis Infor. 2011;4(4):137-140.

86. Jain A, Saxena S, Khanna VK, Shukla RK, Meyer CH. Status of serum VEGF and ICAM-1 and its association with external limiting membrane and inner segment-outer segment junction disruption in type 2 diabetes mellitus. Mol Vis. 2013;19:1760-1768.

87. Uji A, Murakami T, Nishijima K, et al. Association between hyperreflective foci in the outer retina, status of photoreceptor layer, and visual acuity in diabetic macular edema. Am J Ophthalmol. 2012;153(4):710-717, 717.e1.

88. Yanyali A, Bozkurt KT, Macin A, Horozoglu F, Nohutcu AF. Quantitative assessment of photoreceptor layer in eyes with resolved edema after pars plana vitrectomy with internal limiting membrane removal for diabetic macular edema. Ophthalmologica. 2011;226(2): $57-63$.

89. Shin HJ, Lee SH, Chung H, Kim HC. Association between photoreceptor integrity and visual outcome in diabetic macular edema. Graefes Arch Clin Exp Ophthalmol. 2012;250(1):61-70.

90. Chung H, Park B, Shin HJ, Kim HC. Correlation of fundus autofluorescence with spectral-domain optical coherence tomography and vision in diabetic macular edema. Ophthalmology. 2012;119(5):1056-1065.

91. Wakabayashi Y, Kimura K, Muramatsu D, et al. Axial length as a factor associated with visual outcome after vitrectomy for diabetic macular edema. Invest Ophthalmol Vis Sci. 2013;54(10):6834-6840.

92. Ota M, Tsujikawa A, Kita M, et al. Integrity of foveal photoreceptor layer in central retinal vein occlusion. Retina. 2008;28(10):1502-1508.

93. Lima VC, Yeung L, Castro LC, Landa G, Rosen RB. Correlation between spectral domain optical coherence tomography findings and visual outcomes in central retinal vein occlusion. Clin Ophthalmol. 2011;5:299-305.

94. Shin HJ, Chung H, Kim HC. Association between integrity of foveal photoreceptor layer and visual outcome in retinal vein occlusion. Acta Ophthalmol. 2011;89(1):e35-e40.

95. Paunescu LA, Ko TH, Duker JS, et al. Idiopathic juxtafoveal retinal telangiectasis: new findings by ultrahigh-resolution optical coherence tomography. Ophthalmology. 2006;113(1):48-57.

96. Sallo FB, Peto T, Egan C, et al. "En face" OCT imaging of the IS/OS junction line in type 2 idiopathic macular telangiectasia. Invest $\mathrm{Oph}$ thalmol Vis Sci. 2012;53(10):6145-6152.

97. Yannuzzi LA, Bardal AM, Freund KB, Chen KJ, Eandi CM, Blodi B. Idiopathic macular telangiectasia. Arch Ophthalmol. 2006; 124(4):450-460.

98. Charbel Issa P, Gillies MC, Chew EY, et al. Macular telangiectasia type 2. Prog Retin Eye Res. 2013;34:49-77.

99. Matsumoto H, Kishi S, Otani T, Sato T. Elongation of photoreceptor outer segment in central serous chorioretinopathy. Am J Ophthalmol. 2008;145(1):162-168.

100. Kon Y, Iida T, Maruko I, Saito M. The optical coherence tomographyophthalmoscope for examination of central serous chorioretinopathy with precipitates. Retina. 2008;28(6):864-869.

101. Piccolino FC, De La Longrais RR, Manea M, Cicinelli S. Posterior cystoid retinal degeneration in central serous chorioretinopathy. Retina. 2008;28(7):1008-1012.

102. Ojima Y, Hangai M, Sasahara M, et al. Three-dimensional imaging of the foveal photoreceptor layer in central serous chorioretinopathy using high-speed optical coherence tomography. Ophthalmology. 2007;114(12):2197-2207.

103. Piccolino FC, de la Longrais RR, Ravera G, et al. The foveal photoreceptor layer and visual acuity loss in central serous chorioretinopathy. Am J Ophthalmol. 2005;139(1):87-99.
104. Ojima A, Iida T, Sekiryu T, Maruko I, Sugano Y. Photopigments in central serous chorioretinopathy. Am J Ophthalmol. 2011;151(6):940-952.e1.

105. Fujita K, Shinoda K, Imamura Y, et al. Correlation of integrity of cone outer segment tips line with retinal sensitivity after half-dose photodynamic therapy for chronic central serous chorioretinopathy. Am J Ophthalmol. 2012;154(3):579-585.

106. Vasconcelos H, Marques I, Santos AR, et al. Long-term chorioretinal changes after photodynamic therapy for chronic central serous chorioretinopathy. Graefes Arch Clin Exp Ophthalmol. 2013;251(7):1697-1705.

107. Ozdemir O, Erol MK. Morphologic changes and visual outcomes in resolved central serous chorioretinopathy treated with ranibizumab. Cutan Ocul Toxicol. 2014;33(2):122-126.

108. Matsumoto H, Sato T, Kishi S. Outer nuclear layer thickness at the fovea determines visual outcomes in resolved central serous chorioretinopathy. Am J Ophthalmol. 2009;148(1):105-110.e1.

109. Kim SW, Oh J, Huh K. Correlations among various functional and morphological tests in resolved central serous chorioretinopathy. $\mathrm{Br}$ J Ophthalmol. 2012;96(3):350-355.

110. Ojima Y, Tsujikawa A, Hangai M, et al. Retinal sensitivity measured with the micro perimeter 1 after resolution of central serous chorioretinopathy. Am J Ophthalmol. 2008;146(1):77-84.

111. Ozdemir H, Karacorlu SA, Senturk F, Karacorlu M, Uysal O. Assessment of macular function by microperimetry in unilateral resolved central serous chorioretinopathy. Eye (Lond). 2008;22(2):204-208.

112. Aizawa S, Mitamura Y, Baba T, Hagiwara A, Ogata K, Yamamoto S. Correlation between visual function and photoreceptor inner/outer segment junction in patients with retinitis pigmentosa. Eye (Lond). 2009;23(2):304-308

113. Oishi A, Otani A, Sasahara M, et al. Photoreceptor integrity and visual acuity in cystoid macular oedema associated with retinitis pigmentosa. Eye (Lond). 2009;23(6):1411-1416.

114. Sugita T, Kondo M, Piao CH, Ito Y, Terasaki H. Correlation between macular volume and focal macular electroretinogram in patients with retinitis pigmentosa. Invest Ophthalmol Vis Sci. 2008; 49(8):3551-3558.

115. Wakabayashi T, Sawa M, Gomi F, Tsujikawa M. Correlation of fundus autofluorescence with photoreceptor morphology and functional changes in eyes with retinitis pigmentosa. Acta Ophthalmol. 2010;88(5):e177-e183.

116. Iriyama A, Yanagi Y. Fundus autofluorescence and retinal structure as determined by spectral domain optical coherence tomography, and retinal function in retinitis pigmentosa. Graefes Arch Clin Exp Ophthalmol. 2012;250(3):333-339.

117. Aizawa S, Mitamura Y, Hagiwara A, Sugawara T, Yamamoto S. Changes of fundus autofluorescence, photoreceptor inner and outer segment junction line, and visual function in patients with retinitis pigmentosa. Clin Experiment Ophthalmol. 2010;38(6):597-604.

118. Tamaki M, Matsuo T. Optical coherence tomographic parameters as objective signs for visual acuity in patients with retinitis pigmentosa, future candidates for retinal prostheses. J Artif Organs. 2011;14(2): $140-150$.

119. Lima LH, Sallum JM, Spaide RF. Outer retina analysis by optical coherence tomography in cone-rod dystrophy patients. Retina. 2013;33(9): $1877-1880$.

120. Inui E, Oishi A, Oishi M, et al. Tomographic comparison of cone-rod and rod-cone retinal dystrophies. Graefes Arch Clin Exp Ophthalmol. 2014;1-5.

121. Yang P, Michaels KV, Courtney RJ, et al. Retinal Morphology of Patients With Achromatopsia During Early Childhood: Implications for Gene Therapy. JAMA Ophthalmol. 2014.

122. Greenberg JP, Sherman J, Zweifel SA, et al. Spectral-domain optical coherence tomography staging and autofluorescence imaging in achromatopsia. JAMA Ophthalmol. 2014;132(4):437-445.

123. Gomes NL, Greenstein VC, Carlson JN, et al. A comparison of fundus autofluorescence and retinal structure in patients with Stargardt disease. Invest Ophthalmol Vis Sci. 2009;50(8):3953-3959. 
124. Testa F, Rossi S, Sodi A, et al. Correlation between photoreceptor layer integrity and visual function in patients with Stargardt disease: implications for gene therapy. Invest Ophthalmol Vis Sci. 2012; 53(8):4409-4415.

125. Rencova E, Studnicka J, Marak J, Dvorakova H, Langrova H. [The coincidence of the junction layer of inner and outer photoreceptors segments (IS/OS) defects localization on SD OCT and functional defects in case of Stargardt disease - a case report]. Cesk Slov Oftalmol. 2012;68(2):84-86, 88. Czech.

126. Koizumi H, Maguire JI, Spaide RF. Spectral domain optical coherence tomographic findings of occult macular dystrophy. Ophthalmic Surg Lasers Imaging. 2009;40(2):174-176.

127. Kim YG, Baek SH, Moon SW, Lee HK, Kim US. Analysis of spectral domain optical coherence tomography findings in occult macular dystrophy. Acta Ophthalmol. 2011;89(1):e52-e56.

128. Hangai M, Fujimoto M, Yoshimura N. Features and function of multiple evanescent white dot syndrome. Arch Ophthalmol. 2009; 127(10):1307-1313.

129. Nguyen MH, Witkin AJ, Reichel E, et al. Microstructural abnormalities in MEWDS demonstrated by ultrahigh resolution optical coherence tomography. Retina. 2007;27(4):414-418.

130. Tsunoda K, Fujinami K, Miyake Y. Selective abnormality of cone outer segment tip line in acute zonal occult outer retinopathy as observed by spectral-domain optical coherence tomography. Arch Ophthalmol. 2011;129(8):1099-1101.

131. Wolff E. The external limiting membrane of the retina and its relation to Verhoeff's membrane. Trans Ophthalmol Soc U K. 1950;60:61-67.

132. Joussen F, Spitznas M. The fine structure of the human retina at the ora serrata. Albrecht Von Graefes Arch Klin Exp Ophthalmol. 1972;185(3): 177-188.

133. Feeney L. Editorial: Intercellular junctions: sites of permeability barriers and cellular communication. Invest Ophthalmol. 1974;13(11): 811-814.

134. Chen KC, Jung JJ, Alzman A. High definition spectral domain optical coherence tomography findings in three patients with solar retinopathy and review of the literature. Open Ophthalmol J. 2012;6:29-35.

135. Klemencic S, McMahon J, Upadhyay S, Messner L. Spectral domain optical coherence tomography as a predictor of visual function in chronic solar maculopathy. Optom Vis Sci. 2011;88(8):1014-1019.

136. Jain A, Desai RU, Charalel RA, Quiram P, Yannuzzi L, Sarraf D. Solar retinopathy: comparison of optical coherence tomography (OCT) and fluorescein angiography (FA). Retina. 2009;29(9):1340-1345.

137. Chen RW, Gorczynska I, Srinivasan VJ, Fujimoto JG, Duker JS, Reichel E. High-speed ultrahigh-resolution optical coherence tomography findings in chronic solar retinopathy. Retin Cases Brief Rep. 2008;2(2):103-105.
138. Jorge R, Costa RA, Quirino LS, et al. Optical coherence tomography findings in patients with late solar retinopathy. Am J Ophthalmol. 2004;137(6):1139-1143.

139. Iannetti L, Spinucci G, Abbouda A, De Geronimo D, Tortorella P, Accorinti M. Spectral-domain optical coherence tomography in uveitic macular edema: morphological features and prognostic factors. Ophthalmologica. 2012;228(1):13-18.

140. Iannetti L, Tortorella P, D’Ambrosio E, Spena R, Zito R, Gharbiya M. Epiretinal membranes in patients with uveitis: morphological and functional analysis with spectral domain optical coherence tomography. Biomed Res Int. 2013;2013:284821.

141. Gupta V, Gupta A, Dogra MR, Singh I. Reversible retinal changes in the acute stage of sympathetic ophthalmia seen on spectral domain optical coherence tomography. Int Ophthalmol. 2011;31(2): $105-110$.

142. Ishihara K, Hangai M, Kita M, Yoshimura N. Acute Vogt-KoyanagiHarada disease in enhanced spectral-domain optical coherence tomography. Ophthalmology. 2009;116(9):1799-1807.

143. Unoki N, Nishijima K, Kita M, Hayashi R, Yoshimura N. Structural changes of fovea during remission of Behcet's disease as imaged by spectral domain optical coherence tomography. Eye (Lond). 2010; 24(6):969-975.

144. Liu W, Zhang Y, Xu G, Qian J, Jiang C, Li L. Optical coherence tomography for evaluation of photodynamic therapy in symptomatic circumscribed choroidal hemangioma. Retina. 2011;31(2): 336-343.

145. Stepien KE, Han DP, Schell J, Godara P, Rha J, Carroll J. Spectraldomain optical coherence tomography and adaptive optics may detect hydroxychloroquine retinal toxicity before symptomatic vision loss. Trans Am Ophthalmol Soc. 2009;107:28-33.

146. Rodriguez-Padilla JA, Hedges TR, 3rd, Monson B, et al. Highspeed ultra-high-resolution optical coherence tomography findings in hydroxychloroquine retinopathy. Arch Ophthalmol. 2007; 125(6):775-780.

147. Kellner S, Weinitz S, Kellner U. Spectral domain optical coherence tomography detects early stages of chloroquine retinopathy similar to multifocal electroretinography, fundus autofluorescence and near-infrared autofluorescence. Br J Ophthalmol. 2009;93(11): 1444-1447.

148. Kelmenson AT, Brar VS, Murthy RK, Chalam KV. Fundus autofluorescence and spectral domain optical coherence tomography in early detection of Plaquenil maculopathy. Eur J Ophthalmol. 2010;20(4):785-788.

149. Marmor MF, Kellner U, Lai TY, Lyons JS, Mieler WF. Revised recommendations on screening for chloroquine and hydroxychloroquine retinopathy. Ophthalmology. 2011;118(2):415-422.
Clinical Ophthalmology

\section{Publish your work in this journal}

Clinical Ophthalmology is an international, peer-reviewed journal covering all subspecialties within ophthalmology. Key topics include: Optometry; Visual science; Pharmacology and drug therapy in eye diseases; Basic Sciences; Primary and Secondary eye care; Patient Safety and Quality of Care Improvements. This journal is indexed on Submit your manuscript here: http://www.dovepress.com/clinical-ophthalmology-journal

\section{Dovepress}

PubMed Central and CAS, and is the official journal of The Society of Clinical Ophthalmology (SCO). The manuscript management system is completely online and includes a very quick and fair peer-review system, which is all easy to use. Visit http://www.dovepress.com/ testimonials.php to read real quotes from published authors. 